\title{
Vector-borne diseases and their control
}

\author{
Jutta Klasen • Birgit Habedank
}

(C) Springer-Verlag 2008

\section{Introduction}

In the view of climate change, vector-borne diseases of man and animals with vectors and reservoirs developing in natural habitats are of special interest. Vector density, spread of vectors into new geographical areas and the prevalence of infectious agents in the vectors are strongly influenced by micro- and macroclimatic and other environmental conditions. However, climate is only one factor among others: travelling and transportation, human behaviour, socioeconomic conditions, land use and immunity have to be considered for the evaluation of changes in the occurrence of vector-borne diseases (VBD)

Nevertheless, in awareness of forthcoming significant climate change it is necessary to work out the impact to be able to make predictions. Vector and reservoir surveillances are important components of such a strategy.

In 2003, the Federal Environment Agency (FEA) published a report on the 'Possible impact of climate change on distribution of pathogens primarily relevant to humans through animal vectors and to human parasites in Germany' (Maier et al., 2003).

The study compiled all available data on occurrence and distribution of human parasites and disease vectors in Germany until 2001. The overall conclusions were:

- Many of the relationships concerning climate and vectorborne diseases remain postulated ones. Quantitative data are lacking.

- We need studies to close the information gaps about distribution and occurrence of vectors which already exist in Germany and its neighbouring countries.

J. Klasen $(\bowtie) \cdot$ B. Habedank

Federal Environment Agency,

Boetticherstr. 2, Haus 23,

14195 Berlin, Germany

e-mail: Jutta.Klasen@uba.de
Since then, studies form neighbouring countries have also been published which contributed to our current state of knowledge.

In contrast to the great vector-control campaigns on other continents to fight major human infections like malaria, leishmaniasis, trypanosomiasis and onchocerciasis, vectorcontrol programs are not yet common and necessary in Europe. The WHO has reviewed in its reports on vectorborne human infections of Europe (WHO 2004) and on cCASH (Menne and Ebi 2006) that chemical vector-control measures should be limited to application in indoor conditions. Public education and preventive advices are more likely to be effective measures to reduce transmission of VBD in Europe than massive outdoor use of biocides. Multiple interventions that target different points of the disease circle lead to significant decrease in disease transmission, e.g. the consequent destruction and avoidance of breeding places for mosquitoes can reduce transmission rates of mosquito borne diseases significantly. A prerequisite for this strategy is excellent knowledge about biology and ecology of all stages of vector development and the transmission circle.

In many European countries, we are far away from systematic knowledge even about the regional presence of potential disease vectors. The first outbreak of blue-tongue disease in ruminants in Germany in 2006 triggered a first large Midge-Monitoring-System established in March 2007 at 90 German farms, funded by the German Federal Ministry of Food, Agriculture and Consumer Protection. Shall we wait until an epidemic outbreak of a severe human infection, like leishmaniasis or dengue, before we start looking for competent vectors systematically?

Although the vector-borne diseases in Europe do not cause approximately as many deaths than in other continents, we must be prepared for the occurrence of new emerging diseases which can become more likely in a changing environment. 
International networking, interdisciplinary cooperation in research and public education are basic requirements for efficient and productive work in this context.

In September 2007, experts of different disciplines met in the conference 'Vector-Borne Diseases: Impact of Climate Change on Vectors and Rodent Reservoirs' (27 and 28 September 2007 Berlin, Germany). The supplement-in-hand presents articles and abstracts of the contributions, giving an overview of this very interesting and informative conference with many stimulating discussions.

\section{References}

Maier WA, Grunewald J, Habedank B et al. (2003) Possible impact of climate change on distribution of pathogens primarily relevant to humans through animal vectors and to human parasites in Germany. UFOPLAN-Bericht 20061 218/11. Climate Change 05/03. Umweltbundesamt, Berlin, $341 \mathrm{pp}$

Menne B, Ebi KL (Eds) (2006) Climate change and adaptation strategies for human health. Springer Verlag, Steinkopf Verlag Darmstadt, 449 pp

WHO (2004) The vector-borne human infections of Europe-their distribution and burden on public health. WHO Regional Office for Europe, $144 \mathrm{pp}$ 